ZuSATZ. Wenn $d=4 D, D \equiv 2,3(\bmod .4)$ ist, gilt sogar die Ungleichung

$$
h<|d| / 12=|D| / 3,
$$

falls man, abgesehen von dem sowieso ausgeschlossenen Fall $d=-4$, die Körper mit den Diskriminanten $d=-8, d=-20$ und $d=-24$ ausschliesst.

Für alle Diskriminanten $d<-4$ ist also insbesondere der kleinste ganzzahlige positive Rest mod. $p$ von $-C\left((-1)^{\frac{p+1}{2}} m ; \frac{p-1}{2}\right)$ für jede ungerade Primzahl $p$ immer die Klassenzahl selbst, wenn $d \equiv 1(\bmod .4)$ und $m=1$ oder $m=3$ ist, bezw., wenn $d \equiv 0(\bmod .4)$ und $m \leqq 12$ ist.

\title{
LITERATURVERZEICHNIS
}

[1] N. C. Ankeny, E. Artin und S. Chowla, The class-number of real quadratic number fields, Ann. Math. 56 (1952), 479-493.

[2] A. L. Cauchy, Mémoire sur la théorie des nombres. Mémoires de l'Académie royale des sciences de l'Institut de France, t. 17 (1840). Oeuvres, (I), 3, 5-452.

[3] M. GuT, Abschätzungen für die Klassenzahlen der quadratischen Körper. Acta Arithmetica VIII (1963), 113-122.

[4] E. HeCKE, Vorlesungen über die Theorie der algebraischen Zahlen, Leipzig 1923.

[5] A. HuRwIrz, Ueber die Anzahl der Klassen binärer quadratischer Formen von negativer Determinante. Math. Werke Bd. II S. 208 und Acta Mathematica Bd. 19 (1895), 351 und 20 (1897), 312.

Eingegangen den 7. Mai 1966

\section{Zusatz bei der Korrektur:}

Wie ich nachträglich von den Herren A. A. KiseLEV und J. Sch. SLAvUtSKY erfahren habe, enthält eine 1964 in Leningrad erschienene von ihnen verfasste Arbeit (Trudy Cetvertogo Vsesojuznogo Matematičeskogo Sezda, 3.-12. Juli 1961, Bd. II, S. 105-112, Leningrad 1964) nicht nur die Formel (2), sondern für $h$ auch Kongruenzen modulo einer Potenz von $p$ für gewisse Koeffizienten von grösserem als dem Index $(p-1) / 2$ der Reihe auf der rechten Seite von (1). Ihr Resultat ist natürlich mit anderen Mitteln als dem hier benutzten Eulerschen Kriterium gewonnen worden. [M.G.] 